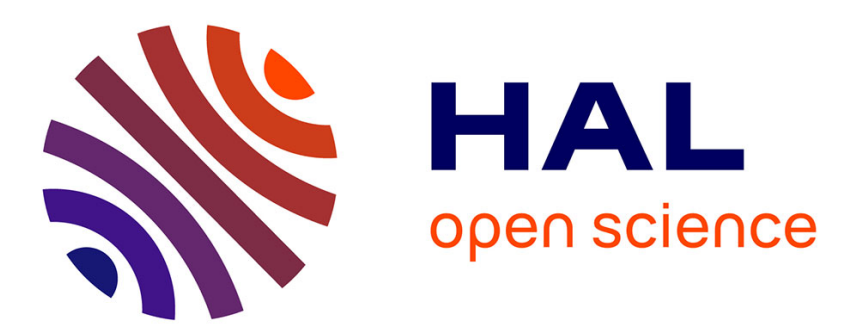

\title{
Segmentation of focal cortical dysplasia lesions on MRI using level set evolution
}

Olivier Colliot, Tommaso Mansi, N. Bernasconi, V. Naessens, D. Klironomos, A. Bernasconi

\section{- To cite this version:}

Olivier Colliot, Tommaso Mansi, N. Bernasconi, V. Naessens, D. Klironomos, et al.. Segmentation of focal cortical dysplasia lesions on MRI using level set evolution. NeuroImage, 2006, 32 (4), pp.16211630. 10.1016/j.neuroimage.2006.04.225 . inria-00614998

\section{HAL Id: inria-00614998 \\ https://inria.hal.science/inria-00614998}

Submitted on 1 Sep 2016

HAL is a multi-disciplinary open access archive for the deposit and dissemination of scientific research documents, whether they are published or not. The documents may come from teaching and research institutions in France or abroad, or from public or private research centers.
L'archive ouverte pluridisciplinaire HAL, est destinée au dépôt et à la diffusion de documents scientifiques de niveau recherche, publiés ou non, émanant des établissements d'enseignement et de recherche français ou étrangers, des laboratoires publics ou privés. 


\section{Segmentation of focal cortical dysplasia lesions on MRI using level set evolution}

O. Colliot, PhD; T. Mansi, MSc; N. Bernasconi, MD, PhD; V. Naessens;

D. Klironomos, MD; A. Bernasconi, MD

Department of Neurology and Neurosurgery and McConnell Brain Imaging Center, Montreal Neurological Institute, McGill University, Montreal, Quebec, Canada

Keywords: epilepsy; focal cortical dysplasia; magnetic resonance imaging; deformable models; level sets

Running Title: Segmentation of focal cortical dysplasia lesions on MRI

\section{Corresponding author:}

Olivier Colliot

McConnell Brain Imaging Center

Montreal Neurological Institute

3801 University Street

Montreal, Quebec, H3A 2B4

Canada

Telephone: (514) 398-3361

Fax: (514) 398-2975

E-mail: colliot@bic.mni.mcgill.ca 


\section{Abstract}

Focal cortical dysplasia (FCD) is the most frequent malformation of cortical development in patients with medically intractable epilepsy. On MRI, FCD lesions are not easily differentiable from the normal cortex and defining their spatial extent is challenging. In this paper, we introduce a method to segment FCD lesions on T1-weighted MRI. It relies on two successive three-dimensional deformable models, whose evolutions are based on the level set framework. The first deformable model is driven by probability maps obtained from three MRI features: cortical thickness, relative intensity and gradient. These features correspond to the visual characteristics of FCD and allow discriminating lesions and normal tissues. In a second stage, the previous result is expanded towards the underlying and overlying cortical boundaries, throughout the whole cortical section. The method was quantitatively evaluated by comparison with manually traced labels in 18 patients with FCD. The automated segmentations achieved a strong agreement with the manuals labels, demonstrating the applicability of the method to assist the delineation of FCD lesions on MRI. This new approach may become a useful tool for the presurgical evaluation of patients with intractable epilepsy related to cortical dysplasia. 


\section{Introduction}

Malformations of cortical development (MCD) (Barkovich et al., 2001) have been increasingly recognized as an important cause of medically intractable focal epilepsy. Focal cortical dysplasia (FCD) (Taylor et al., 1971), a malformation due to abnormal neuroglial proliferation, is the most frequent MCD in patients with intractable extra-temporal epilepsy (Frater et al., 2000; Sisodiya, 2000). Histopathological features of FCD include various degrees of focal cortical thickening, blurring of the gray matter (GM)-white matter (WM) transition, abnormal neuroglial elements in the underlying WM, and disruption of the normal cortical lamination (Palmini et al., 2004; Prayson et al., 2002). Epilepsy surgery, consisting in the resection of the FCD lesion, can be an effective treatment for these patients. However, the prognosis is less favourable (Sisodiya, 2000) than in patients with other causes of intractable epilepsy such as hippocampal sclerosis (Engel, 1996). Poor outcomes may be due to incomplete resection of the lesion (Palmini et al., 1991; Sisodiya, 2000).

Magnetic resonance imaging (MRI) plays a pivotal role in the presurgical evaluation of patients with intractable epilepsy and has allowed the recognition of FCD in an increased number of cases (Barkovich and Kuzniecky, 1996). However, the spatial extension of FCD is difficult to define on MRI as the lesions are often subtle, not easily differentiable from the normal cortex and with ill-defined boundaries. The precise delineation of lesions on MRI could lead to more complete excision and better surgical outcome (Cohen-Gadol et al., 2004; Sisodiya, 2004).

Recently, we (Bernasconi et al., 2001; Antel et al., 2003; Colliot et al., 2005a) and others (Kassubek et al., 2002; Wilke et al., 2003) have developed image analysis techniques to 
detect FCD lesions automatically on MRI, relying on different types of voxel-wise analysis. In particular, computational models of FCD characteristics (Bernasconi et al., 2001; Antel et al., 2002) and a Bayesian classifier for lesion detection (Antel et al., 2003) were previously proposed by our group. While these approaches successfully identify the FCD in a majority of patients, they provide a very limited coverage of the lesions (about 20\%) and cannot be used for their delineation. To our knowledge, the question of FCD segmentation has never been addressed.

Three-dimensional (3D) image segmentation techniques have the potential to provide a reliable and automatic delineation of FCD lesions on MRI. Deformable models are image processing algorithms that make a contour or surface (in 3D) evolve from a starting point, driven by regularization constraints and image data. This approach has been successfully applied to a wide range of medical imaging applications (McInerney and Terzopoulos, 1996), including the reconstruction of the cortex (e.g., Zeng et al., 1999; MacDonald et al., 2000), the segmentation of various subcortical brain structures (e.g., Kelemen et al., 1999; Shen et al., 2002) and of brain tumors (Ho et al., 2002).

This paper presents a method for segmenting FCD lesions on T1-weighted MRI, based on two successive deformable models. The first deformable model is driven by feature maps representing known characteristics of FCD and aims at separating lesions from healthy tissues $^{1}$. The second evolution step expands the result of the first stage towards the underlying and overlying cortical boundaries, troughout the whole cortical section, in order to better cover the full extent of the lesion.

\footnotetext{
${ }^{1}$ A preliminary version of this model was presented in a conference report (Colliot et al., 2005b)
} 


\section{Methods}

\section{Subjects and image preparation}

\section{Subjects}

We studied 24 patients with FCD and partial epilepsy (13 males, mean age \pm [standard deviation $(\mathrm{SD})]=24 \pm 8$ ) whose lesion had been recognized on conventional 3D MRI with multi-planar reformatting. The Ethics Board of the Montreal Neurological Institute and Hospital approved the study, and written informed consent was obtained from all participants.

\section{MRI acquisition}

3D MR images were acquired on a 1.5T scanner (Philips Medical Systems, Best, The Netherlands) using a T1-fast field echo sequence $(T R=18, T E=10,1$ acquisition average pulse sequence, flip angle $=30^{\circ}$, matrix size $=256 \times 256$, FOV $=256$, thickness $\left.=1 \mathrm{~mm}\right)$ with an isotropic voxel size of $1 \mathrm{~mm}^{3}$.

\section{Pre-processing}

All images underwent automated correction for intensity non-uniformity and intensity standardization (Sled et al., 1998) which produces consistent intensities for GM, WM and CSF. They were automatically registered into a common stereotaxic space (Collins et al., 1994) and the brain was separated from the outer layers using the brain extraction tool (BET, Smith, 2002). Classification of brain tissue in GM, WM and CSF was done using an histogram-based method with automated threshold (Antel et al., 2002). 


\section{Overview of the segmentation method}

Our segmentation approach relies on two successive 3D deformable models. A flowchart of the procedure is presented in Figure 1.

The first deformable model, called feature-based deformable model (FDM), aimed at separating the FCD lesion from the normal tissues. It was driven by probability maps obtained from three MR features of FCD. These features were represented using computational models (Antel et al., 2002) and the probabilities were estimated on a training set of lesions. To provide a starting point for the deformable model, we made use of our previously developed FCD classifier (Antel et al., 2003).

The second deformable model expanded the result obtained with the FDM towards the inner and outer cortical boundaries, throughout the whole cortical section. To this purpose, the deformable model was driven by a smooth vector field, computed using the gradient vector flow (GVF) method (Xu and Prince, 1998), pointing towards the boundaries of the cortex. We named this second deformable model "expansion towards cortical boundaries" (ECB).

[Figure 1 about here.]

\section{Feature-based deformable model}

\section{Initialization}

The FCD classifier (Antel et al., 2003) was used under supervision of an expert user to initialize the procedure. The only user intervention that was needed was to verify the output of the classifier. In some cases, the output of the classifier can be composed of several clusters, 
some of them being located outside the FCD lesion. In those cases, the user selected the clusters that co-localized with the lesion which then constituted the starting point of the FDM. The exact nature of these extra-lesional clusters is unknown. Diffuse changes have been previously reported in patients with FCD (Taylor et al., 1971; Prayson et al., 2002; Colliot et al., 2005a) and there is indication that these additional clusters may represent extra-lesional abnormalities (Antel et al., 2003). The behaviour of the deformable model when seeded with an extra-lesional cluster would depend on the size of the cluster. Most likely, small clusters would be shrunk due to the regularization, large ones would be expanded. However, since the aim of this paper is to delineate the primary lesion, the deformable model was initialized with clusters co-localizing with the visual lesion.

\section{Computational models of MRI features of FCD}

On T1-weighted MRI, FCD is characterized by a focal increase of cortical thickness, the presence of hyperintense signal within the dysplastic lesion relative to the normal cortex and a blurred transition between gray and white matter. We previously proposed three computational models that allow to quantitatively evaluate these three abnormal features. In this section, we briefly recall the underlying principles of these models. More details can be found in (Antel et al., 2002).

Cortical thickness was measured by modeling the cortex as an electrostatic field, as proposed by (Jones et al., 2000). A series of isopotential surfaces are generated by solving Laplace's equation over the segmented GM with boundary conditions at the GM/WM and GM/CSF transitions. Thickness at a given voxel is then defined as the length of the path that connects the voxel to both the GM/WM and the GM/CSF transition and is orthogonal 
to all isopotential surfaces. The cortical thickness map was denoted as $T h$.

Hyperintense signal was represented using a relative intensity index defined as $R I(x)=$ $1-\left|B_{g}-I(x)\right| / B_{g}$ where $I(x)$ is the intensity at voxel $x$ and $B_{g}$ is the boundary intensity between GM and WM defined using an automated histogram-based method.

Blurring of the GM/WM transition was modeled with a gradient magnitude map, denoted as $G r$. Prior to computing the gradient, the MRI was convolved with a 3D Gaussian kernel of FWHM=3mm (Full Width at Half Maximum). Areas of blurred GM/WM transition have lower gradient values than areas of normal transition.

These three characteristics defined a vector-valued feature map $f(x)=(\operatorname{Th}(x), R I(x), G r(x))$ at each point $x$ in the image space. Figure 2 (panels B, C and D) presents an example of these feature maps in a patient with FCD.

\section{Probabilistic modeling of FCD features}

We then performed a supervised learning to estimate the probability of different tissue classes in the brain given the feature vector $f$. Six tissue classes were considered: gray matter (GM), white matter (WM), cerebro-spinal fluid (CSF), the transition classes GM/WM and GM/CSF, and the FCD lesion (L). GM, WM and CSF were segmented using a histogrambased approach with automated threshold (Antel et al., 2002) and the FCD lesions were segmented manually on the T1-weighted MRI by a trained observer. The transition between GM and WM was defined by selecting voxels which had a 3x3x3 neighborhood composed of at least $30 \%$ of GM and $30 \%$ of WM, as in the FCD classifier (Antel et al., 2003). The GM/CSF transition was computed using a similar process.

Conditional probabilities $P(f(x) \mid c)$ for each class $c$ were modeled using a trivariate normal 
distribution whose parameters were estimated using the maximum likelihood on a learning set of patients. The posterior probabilities $P(c \mid f(x))$ were then obtained by Bayes' rule. In order to bias the segmentation process towards increased lesion coverage, we assumed equal prior probabilities for the different classes. This empirical choice was supported by our segmentation results which demonstrated that the feature-based deformable model produced very few false positives.

Figure 2 (panels E-J) presents an example of posterior probability maps in a patient with FCD.

[Figure 2 about here.]

\section{Deformable model design}

The feature-based deformable model was designed to separate the lesion from the healthy tissues. The region competition approach proposed by (Zhu and Yuille, 1996) is well adapted to our purpose. This method aims at segmenting an image into several regions by moving the interfaces between them. The evolution of the interfaces is driven by functions indicating the membership of a given voxel to each region. In our case, these functions were derived from the FCD features.

We intended to isolate the FCD lesion from the non-lesional region, which is composed of five different classes (GM, WM, CSF, GM/WM, GM/CSF). However, the boundaries between these five non-lesional classes were of no interest for our application. Thus, region competition occurred in each point between the lesion class and the most probable non-lesional class. The membership to the lesional region was defined as $R_{\mathrm{L}}(x)=P(\mathrm{~L} \mid f(x))$ which is the 
previously computed posterior probability of the lesion class. The non-lesional region was modeled by $R_{\mathrm{NL}}(x)=\max \{P(c \mid f(x)), c \in\{\mathrm{GM}, \mathrm{WM}, \mathrm{CSF}, \mathrm{GM} / \mathrm{WM}, \mathrm{GM} / \mathrm{CSF}\}$.

The feature-based deformable model describes the evolution of the interface (or surface in 3D) $S$ of the lesional region, according to those membership functions and a regularization term. The motion of a point $u$ belonging to $S$ was defined as:

$$
\frac{\partial u}{\partial t}=\alpha_{1}\left[R_{\mathrm{NL}}(u)-R_{\mathrm{L}}(u)\right] \mathbf{n}_{u}+\epsilon_{1} \kappa_{u} \mathbf{n}_{u}
$$

where $\mathbf{n}_{u}$ is the inward normal to $S$ at point $u$ (directed towards the interior of the lesion), $\kappa_{u}$ is the mean curvature and $\alpha_{1}$ and $\epsilon_{1}$ are weighting coefficients.

In the previous equation, $\alpha_{1}\left[R_{\mathrm{NL}}(u)-R_{\mathrm{L}}(u)\right]$ is a feature-based term and $\epsilon_{1} \kappa_{u}$ is a regularity term producing a smooth surface. If $R_{\mathrm{L}}(u)>R_{\mathrm{NL}}(u)$, meaning that the most probable class for point $u$ is the lesion, the surface $S$ is expanded, in order to include this point. On the contrary, if $R_{\mathrm{NL}}(u)>R_{\mathrm{L}}(u)$, meaning that this point should belong to one of the non-lesional classes, the surface is collapsed.

\section{Expansion towards cortical boundaries}

Histological studies have shown that FCD affects all cortical layers (Tassi et al., 2002; Palmini et al., 2004). This indicates that FCD lesions extend from the GM/WM junction, which is the location of the most obvious abnormality on T1-weighted MRI, towards the GM/CSF boundary, over the entire cortical section. This region is not fully covered by the FDM. For this reason, the second deformable model was designed to expand the result of the first stage towards the cortical boundaries, throughout the whole cortical section. On the contrary, 
intra-cortical motion in the lateral direction was prevented in order to avoid progressing into the neighboring healthy cortex.

\section{Expansion with the gradient vector flow}

To drive the deformable model towards the boundaries of the cortex, we relied on a gradient vector flow (GVF) (Xu and Prince, 1998). In brief, the GVF is computed by diffusion of the gradient vector and provides a smooth vector field which is approximately orthogonal to the GM boundaries. We computed the GVF from the GM segmentation and not directly from the MRI because FCD are characterized by a blurring of the GM/WM transition. Thus, a gradient computed directly from the original image would have a too small magnitude to adequately drive the deformable model. The GM segmentation was first slightly smoothed with a 3D Gaussian kernel (FWHM=2mm). An edge map $f$ was then obtained by computing the norm of the gradient of the previous result. The GVF was defined as the equilibrium solution of the following equation:

$$
\left\{\begin{array}{l}
\frac{\partial \mathbf{v}}{\partial t}=g(\|\nabla f\|) \nabla^{2} \mathbf{v}-h(\|\nabla f\|)(\mathbf{v}-\nabla f) \\
\mathbf{v}(x, 0)=\nabla f(x)
\end{array}\right.
$$

where $\nabla^{2}$ is the Laplacian operator, $g(r)$ and $h(r)$ are weighting functions defined as $g(r)=$

$e^{-\frac{r}{K}}$ and $h(r)=1-g(r)$, and $K$ is a positive constant which controls the amount of smoothing. If $K$ is high, the diffusion term $\nabla^{2} \mathbf{v}$ prevails and the resulting vector field is smoother. Conversely, low values of $K$ produce a GVF closer to the original gradient. Finally, the GVF was normalized: $\hat{\mathbf{v}}(u)=\mathbf{v}(u) /\|\mathbf{v}(u)\|$. An example of GVF is shown in 
Figure 3 .

[Figure 3 about here.]

The following force was then used to drive the deformable model towards the cortical boundaries using the GVF:

$$
\mathbf{F}_{G V F}=\left[\hat{\mathbf{v}}(u) \cdot \mathbf{n}_{u}\right] \mathbf{n}_{u}
$$

where $\mathbf{n}_{u}$ is the inward normal to the surface at point $u, \hat{\mathbf{v}}(u)$ is the normalized GVF and . is the scalar product.

This force enables the model to progress in a direction orthogonal to the cortical boundaries while avoiding lateral motion. When the normal to the surface is oriented along the GVF, $F=\left\|\mathbf{F}_{G V F}\right\|>0$ and the surface is attracted towards the boundaries of GM. Conversely, when the normal is orthogonal to the GVF, $F=0$ which prevents the model from expanding laterally into the neighboring cortex. This is illustrated in Figure 4.

[Figure 4 about here.]

\section{Deformable model design}

The second deformable model, which is called "expansion towards cortical boundaries" (ECB), combines FCD features with the GVF motion. The GVF is used to expand the result obtained with the previous FDM, towards the boundaries of the cortex. The MR features restrict the GVF motion, to prevent the deformable model from progressing into healthy regions. Moreover, the GVF is not taken into account in points which possess MR 
features of FCD. This ensures that lesional regions segmented by the FDM will not be shrunk by the second deformable model.

The motion of a point $u$ of the interface $S$ was then defined as:

$$
\frac{\partial u}{\partial t}=\alpha_{2}\left[R_{\mathrm{NL}}(u)-R_{\mathrm{L}}(u)\right] \mathbf{n}_{u}+\beta_{2} \delta(u)\left[\hat{\mathbf{v}}(u) \cdot \mathbf{n}_{u}\right] \mathbf{n}_{u}+\epsilon_{2} \kappa_{u} \mathbf{n}_{u}
$$

where $\delta(u)=1$ if $R_{\mathrm{NL}}(u)>R_{\mathrm{L}}(u)$ and $\delta(u)=0$ if $R_{\mathrm{NL}}(u) \leq R_{\mathrm{L}}(u), \alpha_{2}, \beta_{2}$ and $\epsilon_{2}$ are weighting coefficients and $\kappa_{u}, \mathbf{n}_{u}$ and $\hat{\mathbf{v}}(u)$ are defined as in Equations 1 and 3.

$\left[R_{\mathrm{NL}}(u)-R_{\mathrm{L}}(u)\right] \mathbf{n}_{u}$ is the feature-based term. $\delta(u)\left[\hat{\mathbf{v}}(u) \cdot \mathbf{n}_{u}\right] \mathbf{n}_{u}$ is the GVF-based force defined in Equation 3 and is canceled if $R_{\mathrm{L}}(u) \geq R_{\mathrm{NL}}(u)$, i.e. if point $u$ possesses the MR features of FCD.

Figure 5 presents an example of the different steps of the evolution.

[Figure 5 about here.]

\section{Level set evolution}

The motion equations 1 and 4 of the two deformable models were both implemented using the level set method (Osher and Sethian, 1988; Sethian, 1999). The principle of this method is to define the surface $S$ as the zero level set of a higher dimensional function $\phi$, called the implicit function:

$$
\phi(S(t), t)=0
$$


As an implicit function $\phi$, we chose the classical signed distance to the surface $S$, with negative values in the interior of $S$. The evolution was then performed on the function $\phi$ and the embedded surface $S$ was deformed implicitly. Level sets offer several advantages over traditional deformable models: no parameterization of the surface is necessary, topology changes are handled naturally and the result is less sensitive to the initialization.

Using the derivation from curve motion to level set evolution (Sethian, 1999), the featurebased deformable model was described by:

$$
\frac{\partial \phi}{\partial t}(x)=\alpha_{1}\left[R_{\mathrm{NL}}(x)-R_{\mathrm{L}}(x)\right]|\nabla \phi(x)|+\epsilon_{1} \kappa_{x}|\nabla \phi(x)|
$$

Similarly, the evolution towards cortical boundaries was performed according to:

$$
\frac{\partial \phi}{\partial t}(x)=\alpha_{2}\left[R_{\mathrm{NL}}(x)-R_{\mathrm{L}}(x)\right]|\nabla \phi(x)|-\beta_{2} \delta(x)[\hat{\mathbf{v}}(x) \cdot \nabla \phi(x)]+\epsilon_{2} \kappa_{x}|\nabla \phi(x)|
$$

The two previous equations were implemented using the numerical scheme proposed in (Sethian, 1999, chap.6). To reduce the computational complexity, we made use of the narrow-band method (Adalsteinsson and Sethian, 1995). A sub-voxel reinitialization of the implicit function was performed at fixed time steps to maintain the distance function (Krissian and Westin, 2005). 


\section{Evaluation}

\section{Manual segmentations}

Lesions were delineated independently on 3D MRI by two trained raters (VN and DK) using the interactive software package DISPLAY developed at the Brain Imaging Center of the Montreal Neurological Institute. This program allows simultaneous viewing of MR images in coronal, sagittal and horizontal orientations. The corresponding manually labeled datasets are further denoted as $M_{1}$ and $M_{2}$. Inter-rater agreement was assessed using the similarity

index $S=2 \frac{\left|M_{1} \cap M_{2}\right|}{\left|M_{1}\right|+\left|M_{2}\right|}$ (where $|M|$ is the number of elements in set $M$ ), which is a special case of kappa statistic since the vast majority of voxels are non-lesional (Zijdenbos et al., 1994).

However, the evaluation of the automated segmentation is limited by the differences between the two manual labels. To overcome this difficulty, we proposed to build "consensus" manual labels, denoted as $M_{C}$. To this purpose, two other observers (NB and AB) jointly inspected the tracings $M_{1}$ and $M_{2}$ of the two raters and provided a decision for all ambiguous regions (i.e., regions defined as lesional by one rater but not by the other).

\section{Level set segmentations}

The level set segmentations were obtained as follows. First, the FCD classifier was used to obtain an initialization for the FDM. The intermediate result obtained with the FDM then constituted the starting point of the ECB whose output defined the final FCD segmentation. We used the following segmentation parameters: $\alpha_{1}=0.8$ and $\epsilon_{1}=0.2$ for the FDM; $\alpha_{2}=0.2, \beta_{2}=0.8$ and $\epsilon_{2}=0.1$ in the ECB. No fine tuning of the parameters was necessary and the same values were used for all subjects. 
The evaluation was performed using a leave-one-out approach: for the segmentation of a given patient, this patient was excluded from the learning set. This approach avoids the introduction of bias in the result.

The performance of each of step of the procedure (classifier, FDM and ECB) was assessed by comparison with the "consensus labels" $M_{C}$, using the following metrics:

- the aforementioned similarity index $S=2 \frac{\left|A \cap M_{C}\right|}{|A|+\left|M_{C}\right|}$ (where $A$ is the automated segmentation);

- a coverage index $C=100 \times\left|A \cap M_{C}\right| /\left|M_{C}\right|$ which indicates the percentage of voxels in the manual label that were successfully classified as lesional by the level set;

- a false positive index $F P=100 \times\left|A \backslash M_{C}\right| /|A|$ (where $\backslash$ denotes the set subtraction), which indicates the percentage of voxels that were wrongly classified as lesional by the level set.

The probabilistic modeling of FCD features is based on a training on manually labeled lesions. To assess the influence of the training dataset on the segmentation, we compared the similarity indices obtained using a learning on labels $M_{1}$ to those obtained using labels $M_{2}$.

\section{Results}

\section{Initialization}

The FCD classifier (Antel et al., 2003) was used to initialize the FDM. It successfully identified the lesion in $18(18 / 24=75 \%)$ patients. The evaluation was thus done on the 18 detected 
lesions.

\section{Manual segmentations}

For the 18 manual labels, the mean inter-rater similarity index was $0.62 \pm 0.19$ (range $=0.22$ to 0.84$)$.

\section{Level set segmentation}

The similarity, coverage, and false positive indices obtained at each step of the procedure (final result with the ECB, intermediate result of the FDM and initialization with the classifier) compared to the consensus manual labels $M_{C}$, in the 18 detected cases, are reported in Table 1. Segmentation results in five patients with FCD are shown in Figure 6. Some examples of 3D renderings of the lesion segmentation are presented in Figure 7.

[Table 1 about here.]

[Figure 6 about here.]

[Figure 7 about here.]

The similarity indices obtained with a training based on labels $M_{1}$ were: $0.73 \pm 0.08$ (range $=0.60$ to 0.86$)$ (the values are the same as in Table 1 where all results were obtained using $M_{1}$ as a learning set). Those obtained with $M_{2}$ were: $0.72 \pm 0.09$ (range $=0.56$ to 0.85). All similarities were computed using $M_{C}$ as a reference. 


\section{Discussion}

In this study, we proposed and evaluated a method to segment FCD lesions on T1-weighted MRI, relying on two successive deformable models. We first introduced a feature-based level set, driven by known MR characteristics of FCD, which separates lesions from non-lesional tissue. The second deformable model was designed to expand the previous result towards the cortical boundaries while preventing lateral intra-cortical motion into healthy tissues.

\section{Segmentation results}

The level set segmentations achieved a degree of similarity of 0.73 with the consensus manual labels, which constitutes a strong agreement (Zijdenbos et al., 1994). The mean false positive index was 20\%, which constitutes a low value when dealing with small structures such as FCD lesions. Indeed, small objects are penalized by this measure since a minor segmentation error can lead to a substantial percentage of false positives. For example, if we consider as reference object a sphere with a volume equal to the mean volume of FCD lesions in our patients $\left(16.8 \mathrm{~cm}^{3}\right)$, and as segmentation result a sphere whose radius is one voxel longer, we obtain $F P=17 \%$. In some cases, we noted that a narrow sulcus, located near the boundary of the lesion, was not correctly classified as CSF and, as a consequence, part of the neighboring healthy gyrus was included in the lesion segmentation (an example is presented in Figure 8). Nevertheless, the generated false positives were generally small and easily identifiable. They did not penalize the overall performance of the automated segmentation.

These results demonstrated good accuracy of our segmentation approach which, when combined with additional manually traced corrections, could be a useful tool to assess the 
extent of FCD lesions on MRI. By being objective and reproducible, this technique can overcome the limits of manual tracing and provide more reliable delineation. Moreover, since this method operates in $3 \mathrm{D}$, it has the potential to unveil lesional areas that could be overlooked due to the complexity of the cortex convolution.

[Figure 8 about here.]

Table 1 also shows the improvement of the second deformable model over the FDM. It provided a substantial enhancement of the lesion coverage with only a small increase of false positives, resulting in a stronger overall similarity.

When using two different sets of manual labels for the training step, the results of the automated segmentation were almost identical. This shows the robustness of our method with respect to the learning dataset. Moreover, no fine-tuning of the level set parameters was necessary and the same parameters were used for all patients. Furthermore, the method is fast thanks to the use of the narrow-band and the subvoxel reinitialization approaches (the mean computation time for the level set evolution was 14 minutes on a PC $1.6 \mathrm{GHz}$ ).

\section{Comparison with other image analysis techniques}

So far, image analysis methods in FCD have mostly relied on voxel-wise techniques (Wilke et al., 2003; Antel et al., 2003; Colliot et al., 2005a). A Bayesian classifier based on computational models and texture features was previously proposed by our group (Antel et al., 2003). We recently introduced an individual VBM analysis that was able to detect both primary lesions and extra-lesional abnormalities (Colliot et al., 2005a). These techniques allow the identification of subtle FCD lesions and are therefore useful to assist the clinical 
diagnosis. However, they recover only a small fraction of the lesional area (between $15 \%$

and 30\%). By using an image segmentation approach based on deformable models, we were able to automatically detect over $70 \%$ of lesional voxels. To our knowledge, this is the first quantitative method that can be used to assess the spatial extent of FCD lesions.

\section{Evaluation methodology}

There is no available gold standard for evaluating the delineation of FCD lesions. On MRI, these lesions possess ill-defined contours and are not easily differentiable from normal cortex. Their tracing by experts necessarily suffers from subjectivity. For these reasons, we aimed at pooling the knowledge of several raters to provide a more objective reference than if separate manual labels were used. First, manual tracings were done separately by two raters. The inter-rater similarity was 0.62 which corresponds to a substantial agreement, in particular when keeping in mind the difficulty of FCD segmentation. To reduce the remaining variability, "consensus" labels were designed with the help of two additional experts.

\section{Initialization}

To provide a starting point for the segmentation procedure, we relied on our previously developed Bayesian FCD classifier (Antel et al., 2003), under supervision of an expert user. This semi-automated initialization allows keeping user intervention to a minimal level and reducing subjectivity. However, the classifier failed to detect six FCD lesions. When providing a manual initialization to the FDM in those six cases, the deformable surface shrunk and the segmentation failed. The inspection of the feature maps and the probability maps revealed 
that the three features were not discriminating enough to adequately drive the deformable model. Nevertheless, FCD lesions are difficult to detect and our detection rate is similar to those reported for other methods (Wilke et al., 2003). Moreover, our segmentation approach was applicable in $75 \%$ of cases and can thus be a useful tool for the presurgical evaluation of a large number of patients with FCD.

\section{Clinical considerations}

For our experiments, we selected patients with FCD whose lesion had been recognized preoperatively on 3D MRI with multi-planar reformatting. We thus excluded patients whose lesion could not be recognized prior to surgery. The rationale for this criterion was that, in a presurgical setting, the assessment of the lesion extent is done after the FCD has been seen on the MRI. The aim of this paper was to assist the delineation of the lesional area once the FCD has been diagnosed. Thus, in its present form, the segmentation algorithm should only be applied to MRI-visible FCDs.

The patient group included FCDs of various sizes, with volumes spanning from $1.3 \mathrm{~cm}^{3}$ to $94.6 \mathrm{~cm}^{3}$ (as defined by the "consensus" manual labels). The segmentation algorithm behaved similarly with small and large lesions, indicating that potential difficulties in delineating FCD lesions are not necessarily linked to their size. Moreover we did not observe any difference in terms of segmentation accuracy depending on the region of the brain where the lesion was located.

In terms of image quality, our segmentation algorithm has requisites similar to those of other quantitative image analysis techniques. The MRI sequence should offer a strong 
gray-white contrast and thin slices, to be able to determine precisely the cortical boundaries. In our previous work, we evaluated the images generated by our protocol and found a high signal-to-noise ratio (Antel et al., 2002). We also verified that all our MRIs were free of visible motion artefacts. In this study, all the MR images were acquired on a $1.5 \mathrm{~T}$ scanner using a standard T1-fast field echo sequence. Our segmentation approach is thus applicable in a real world context since this type of MRI acquisition is available in a clinical context.

In conclusion, this paper demonstrates the effectiveness of a deformable model approach for the segmentation of FCD lesions. This new method can be used to help assessing the spatial extent of FCD lesions on MRI and has the potential to unveil lesional areas that could be overlooked by visual inspection. It may become a useful tool for surgical planning in patients with cortical dysplasia.

\section{Acknowledgments}

This work was supported by a grant of the Canadian Institutes of Health Research (CIHR grant \#203707) and by the Scottish Rite Charitable Foundation of Canada. O.C. is recipient of the Epilepsy Canada Clinical Sciences Fellowship. The authors are grateful to Dr F. Andermann and F. Dubeau for referring patients to the study. 


\section{References}

Adalsteinsson, D., Sethian, J., 1995. A fast level set method for propagating interfaces. Journal of Computational Physics , 269-277.

Antel, S., Bernasconi, A., Bernasconi, N., Collins, D., Kearney, R., Shinghal, R., Arnold, D., 2002. Computational models of MRI characteristics of focal cortical dysplasia improve lesion detection. NeuroImage 17 (4), 1755-60.

Antel, S., Collins, D., Bernasconi, N., Andermann, F., Shinghal, R., Kearney, R., Arnold, D., Bernasconi, A., 2003. Automated detection of focal cortical dysplasia lesions using computational models of their MRI characteristics and texture analysis. NeuroImage 19 (4), $1748-59$.

Barkovich, A., Kuzniecky, R., 1996. Neuroimaging of focal malformations of cortical development. J Clin Neurophysiol 13 (6), 481-494.

Barkovich, A. J., Kuzniecky, R. I., Jackson, G. D., Guerrini, R., Dobyns, W. B., 2001. Classification system for malformations of cortical development: update 2001. Neurology 57 (12), 2168-78.

Bernasconi, A., Antel, S., Collins, D., Bernasconi, N., Olivier, A., Dubeau, F., Pike, G., Andermann, F., Arnold, D., 2001. Texture analysis and morphological processing of magnetic resonance imaging assist detection of focal cortical dysplasia in extra-temporal partial epilepsy. Ann Neurol 49 (6), 770-5. 
Cohen-Gadol, A., Ozduman, K., Bronen, R., Kim, J., Spencer, D., 2004. Long-term outcome after epilepsy surgery for focal cortical dysplasia. J Neurosurg 101, 55-65.

Collins, D., Neelin, P., Peters, T., Evans, A., 1994. Automatic 3D intersubject registration of MR volumetric data in standardized Talairach space. J Comput Assist Tomogr 18, $192-205$.

Colliot, O., Bernasconi, N., Khalili, N., Antel, S., Naessens, V., Bernasconi, A., $2005 a$. Individual voxel-based analysis of gray matter in focal cortical dysplasia. NeuroImage (In Press).

Colliot, O., Mansi, T., Bernasconi, N., Naessens, V., Klironomos, D., Bernasconi, A., 2005 b. Segmentation of focal cortical dysplasia lesions using a feature-based level set. In: Duncan, J., Gerig, G. (Eds.), Proc. Medical Image Computing and Computer-Assisted Intervention MICCAI 2005. Vol. 3749 of Lecture Notes in Computer Science. Springer, Palm Springs, CA, USA, pp. 375-382.

Engel, J., 1996. Surgery for seizures. N Engl J Med 334 (10), 647-652.

Frater, J., Prayson, R., Morris III, H., Bingaman, W., 2000. Surgical pathologic findings of extratemporal-based intractable epilepsy: a study of 133 consecutive resections. Arch Pathol Lab Med 124 (4), 545-549.

Ho, S., Bullit, E., Gerig, G., 2002. Level-set evolution with region competition: automatic 3-D segmentation of brain tumors. In: Proc. International Conference on Pattern Recognition ICPR 2002. Vol. 1. pp. 532-535. 
Jones, S., Buchbinder, B., Aharon, I., 2000. Three-dimensional mapping of cortical thickness using Laplace's equation. Hum Brain Mapp 11 (1), 12-32.

Kassubek, J., Huppertz, H., Spreer, J., Schulze-Bonhage, A., 2002. Detection and localization of focal cortical dysplasia by voxel-based 3-D MRI analysis. Epilepsia 43, 596-602.

Kelemen, A., Szekely, G., Gerig, G., 1999. Elastic model-based segmentation of 3D neuroradiological data sets. IEEE Trans Med Imaging 18 (10), 828-839.

Krissian, K., Westin, C.-F., 2005. Fast sub-voxel re-initialization of the distance map for level set methods. Pattern Recognition Letters 26, 1532-1542.

MacDonald, D., Kabani, N., Avis, D., Evans, A., 2000. Automated 3D extraction of inner and outer surfaces of cerebral cortex from MRI. NeuroImage 12 (3), 340-356.

McInerney, T., Terzopoulos, D., 1996. Deformable models in medical image analysis: a survey. Med Image Analysis 1 (2), 91-108.

Osher, S., Sethian, J., 1988. Fronts propagating with curvature-dependent speed : algorithms based on Hamilton-Jacobi formulation. Journal of Computational Physics 79, 12-49.

Palmini, A., Andermann, F., Olivier, A., Tampieri, D., Robitaille, Y., Andermann, E., et al., 1991. Focal neuronal migration disorders and intractable partial epilepsy: a study of 30 patients [Review]. Ann Neurol 30, 741-9.

Palmini, A., Najm, I., Avanzini, G., Babb, T., Guerrini, R., Foldvary-Schæfer, N., Jackson, G., Lüders, H., Prayson, R., Spreafico, R., Vinters, H., 2004. Terminology and classification of the cortical dysplasias. Neurology 62, S2-S8. 
Prayson, R., Spreafico, R., Vinters, H., 2002. Pathologic characteristics of the cortical dysplasias. Neurosurg Clin N Am 13 (1), 17-25.

Sethian, J., 1999. Level-set methods and fast marching methods, 2nd Edition. Cambridge University Press.

Shen, D., Moffat, S., Resnick, S., Davatzikos, C., 2002. Measuring size and shape of the hippocampus in MR images using a deformable shape model. NeuroImage 15, 422-434.

Sisodiya, S., 2000. Surgery for malformations of cortical development causing epilepsy. Brain 123, 1075-1091.

Sisodiya, S., 2004. Surgery for focal cortical dysplasia. Brain 127, 2383-4.

Sled, J., Zijdenbos, A., Evans, A., 1998. A nonparametric method for automatic correction of intensity nonuniformity in MRI data. IEEE Trans Med Imaging 17 (1), 87-97.

Smith, S., Nov. 2002. Fast robust automated brain extraction. Human Brain Mapping 17 (3), $143-155$.

Tassi, L., Colombo, N., Garbelli, R., Francione, S., Lo Russo, G., Mai, R., Cardinale, F., Cossu, M., Ferrario, A., Galli, C., Bramerio, M., Citterio, A., Spreafico, R., 2002. Focal cortical dysplasia: neuropathological subtypes, EEG, neuroimaging and surgical outcome. Brain 125 (Pt 8), 1719-1732.

Taylor, D., Falconer, M., Bruton, C., Corsellis, J., 1971. Focal dysplasia of the cerebral cortex in epilepsy. J Neurol Neurosurg Psychiatry 34, 369-387. 
Wilke, M., Kassubek, J., Ziyeh, S., Schulze-Bonhage, A., Huppertz, H., 2003. Automated detection of gray matter malformations using optimized voxel-based morphometry: a systematic approach. NeuroImage 20 (1), 330-343.

Xu, C., Prince, J., 1998. Snakes, shapes and gradient vector flow. IEEE Trans Image Proc $7(3), 359-369$.

Zeng, X., Staib, L., Schultz, R., Duncan, J., 1999. Segmentation and measurement of the cortex from 3-D MR images using coupled-surfaces propagation. IEEE Trans Med Imaging $18(10), 927-37$.

Zhu, S., Yuille, A., 1996. Region competition: Unifying snakes, region growing, and bayes/MDL for multiband image segmentation. IEEE Trans Pattern Anal Mach Intell $18(9), 884-900$.

Zijdenbos, A., Dawant, B., Margolin, R., Palmer, A., 1994. Morphometric analysis of white matter lesions in MR images: method and validation. IEEE Trans Med Imaging 13 (4), $716-724$. 


\section{List of Tables}

1 Quantitative evaluation of the automated segmentation. The table presents the evaluation of the final result $(\mathrm{ECB})$ and of the different steps in the procedure (FDM, classifier). For each step, the similarity index $S$, the coverage index $C$ and the false positive index $F P$ were computed with respect to the consensus labels $M_{C}$. Each cell is shown as mean $\pm \mathrm{SD}$ with the range in parentheses. 
Table 1: Quantitative evaluation of the automated segmentation. The table presents the evaluation of the final result (ECB) and of the different steps in the procedure (FDM, classifier). For each step, the similarity index $S$, the coverage index $C$ and the false positive index $F P$ were computed with respect to the consensus labels $M_{C}$. Each cell is shown as mean $\pm \mathrm{SD}$ with the range in parentheses.

\begin{tabular}{|c|c|c|c|}
\hline & $S$ & $C$ & $F P$ \\
\hline ECB & $0.73 \pm 0.08(0.60$ to 0.86$)$ & $72 \% \pm 16 \%(44 \%$ to $94 \%)$ & $20 \% \pm 15 \%(0.4 \%$ to $48 \%)$ \\
\hline FDM & $0.57 \pm 0.17(0.22$ to 0.77$)$ & $45 \% \pm 18 \%(12 \%$ to $71 \%)$ & $9 \% \pm 10 \%(0 \%$ to $31 \%)$ \\
Classifier & $0.26 \pm 0.14(0.05$ to 0.46$)$ & $16 \% \pm 10 \%(3 \%$ to $30 \%)$ & $0.3 \% \pm 0.8 \%(0 \%$ to $3.1 \%)$ \\
\hline
\end{tabular}




\section{List of Figures}

1 Flowchart summarizing the different stages of the segmentation procedure. Our previously developed FCD classifier is used to provide an initialization to the first deformable model. This feature-based deformable model (FDM) is driven by probability maps of FCD features which are estimated from a training set of patients. The intermediate result obtained with the FDM is then expanded towards the cortical boundaries by using a gradient vector flow $(\mathrm{GVF}) \ldots \ldots \ldots \ldots \ldots \ldots \ldots$

2 Probabilistic modeling of FCD features. The upper row shows: the T1weighted MRI where the FCD lesion is indicated by the arrow $(\mathrm{A})$, the cortical thickness map (B), the relative intensity map (C) and the gradient map (D). The lesion is characterized by higher cortical thickness, higher relative intensity and lower gradient. The two lower rows show the probability maps of the lesion class (E), GM (F), WM (G), CSF (H), the transition between GM and WM (I) and the transition between GM and CSF (J). . . . . . . . .

3 Computation of the gradient vector flow $(\mathrm{GVF})$ in a patient with FCD. (A) GVF computed from the edge map. (B) Detail of the normalized GVF. . 4 Expansion towards the boundaries of the cortex using the GVF. When the normal to the surface is oriented along the GVF (indicated by the gray arrows), $F>0$ and the deformable model is expanded towards the GM/WM and GM/CSF interfaces. On the contrary, when the normal is orthogonal to the GVF, $F=0$ which prevents lateral motion into the neighboring cortex. . .

5 Example of FCD lesion segmentation using the two successive deformable models. (A) T1-weighted MRI where the FCD lesion is indicated by an arrow. (B) Final result shown together with the gradient vector flow (GVF). (C) Initialization with the FCD classifier. (D) Intermediate result of the feature-based deformable model (FDM). (E) Final result using the expansion towards cortical boundaries $(\mathrm{ECB}) . \ldots \ldots \ldots$

6 Results of the lesion segmentation in five different subjects. Columns show the different steps of the procedure and the corresponding manual label: (A) initialization with the classifier (yellow contour), (B) intermediate result with the FDM (red contour), (C) final result of the automated segmentation with the ECB (red contour), (D) consensus manual label $M_{C}$ (blue contour). . . . $3 \mathrm{D}$ renderings of the automated lesion segmentation in three patients with FCD, shown together with the cortical surface. Visualization was done using the BrainVISA/Anatomist software (www.brainvisa.info). . . . . . . . . . . .

8 Example of a narrow sulcus which was not correctly extracted. (A) T1weighted MRI where the sulcus (indicated by the arrow) is located near the inferior boundary of the FCD lesion. (B) Segmentation result. The deformable model has progressed beyond the sulcus resulting in a false positive area. (C) Manually traced lesion label $M_{C}$. 


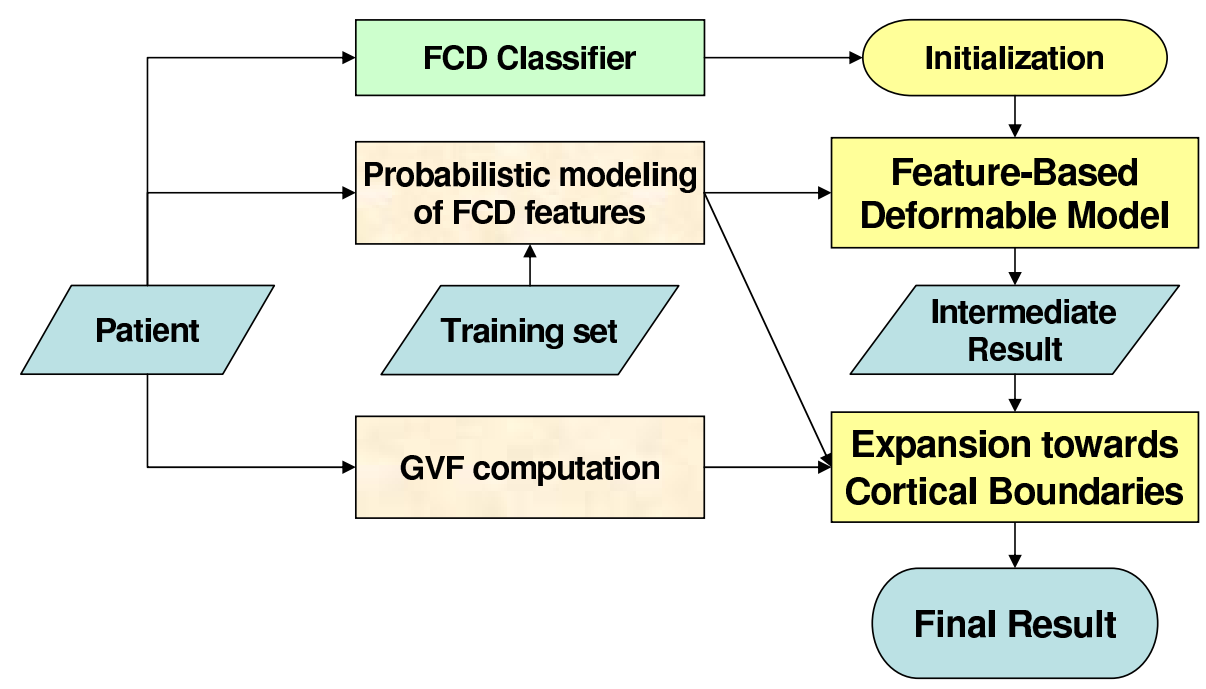

Figure 1: Flowchart summarizing the different stages of the segmentation procedure. Our previously developed FCD classifier is used to provide an initialization to the first deformable model. This feature-based deformable model (FDM) is driven by probability maps of FCD features which are estimated from a training set of patients. The intermediate result obtained with the FDM is then expanded towards the cortical boundaries by using a gradient vector flow $(\mathrm{GVF})$. 

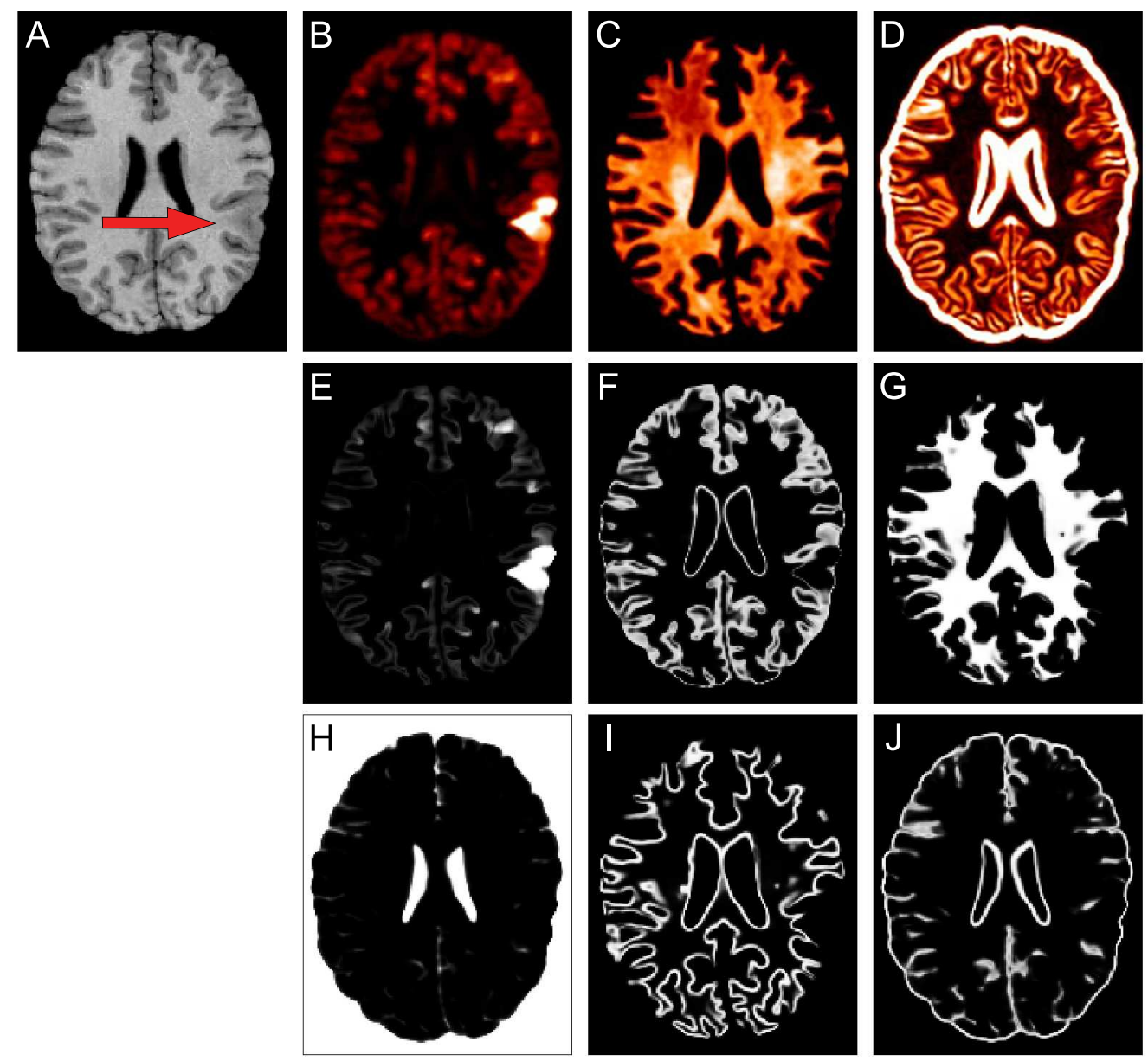

Figure 2: Probabilistic modeling of FCD features. The upper row shows: the T1-weighted MRI where the FCD lesion is indicated by the arrow (A), the cortical thickness map (B), the relative intensity map (C) and the gradient map (D). The lesion is characterized by higher cortical thickness, higher relative intensity and lower gradient. The two lower rows show the probability maps of the lesion class (E), GM (F), WM (G), CSF (H), the transition between GM and WM (I) and the transition between GM and CSF (J). 


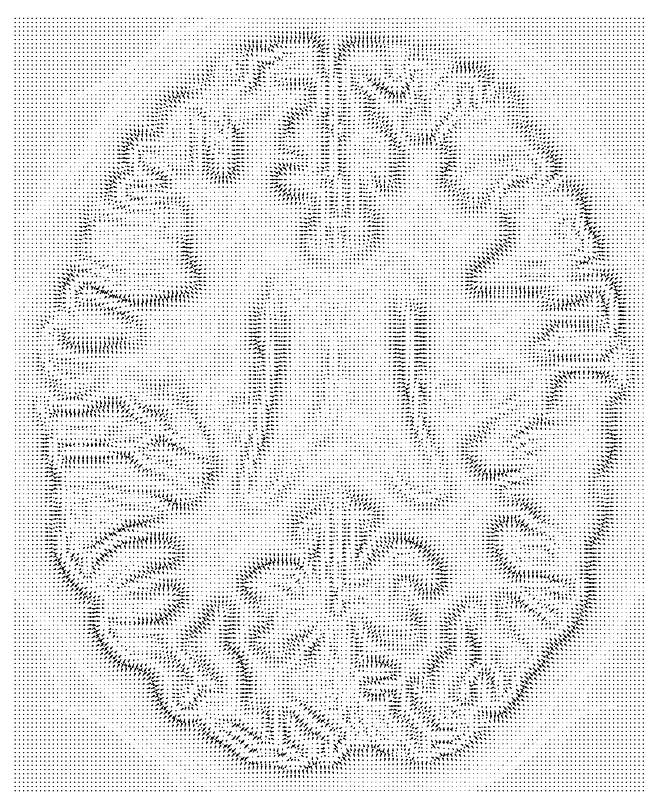

A

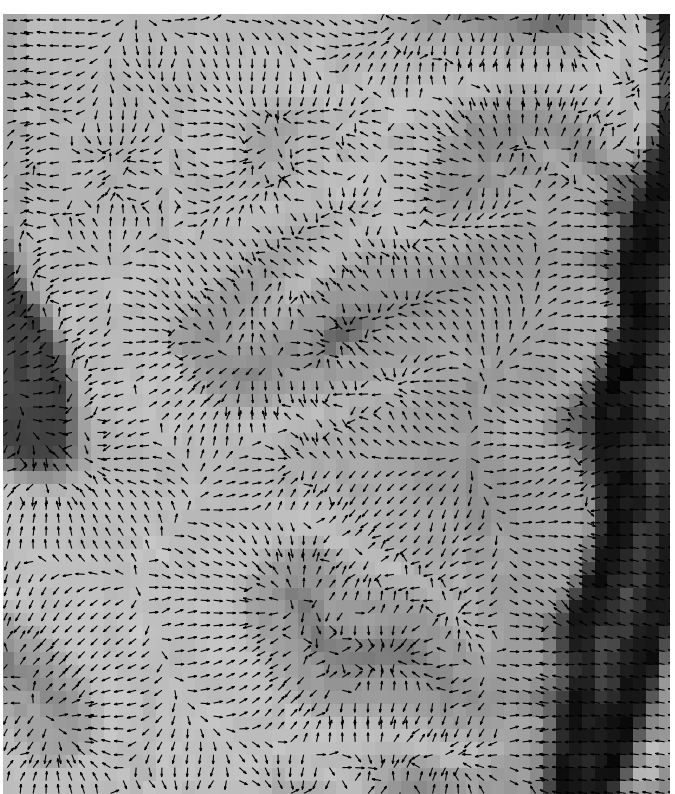

B

Figure 3: Computation of the gradient vector flow (GVF) in a patient with FCD. (A) GVF computed from the edge map. (B) Detail of the normalized GVF. 


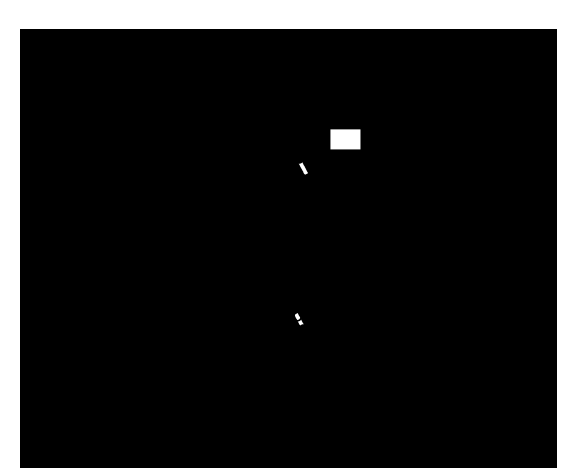

Figure 4: Expansion towards the boundaries of the cortex using the GVF. When the normal to the surface is oriented along the GVF (indicated by the gray arrows), $F>0$ and the deformable model is expanded towards the GM/WM and GM/CSF interfaces. On the contrary, when the normal is orthogonal to the GVF, $F=0$ which prevents lateral motion into the neighboring cortex. 

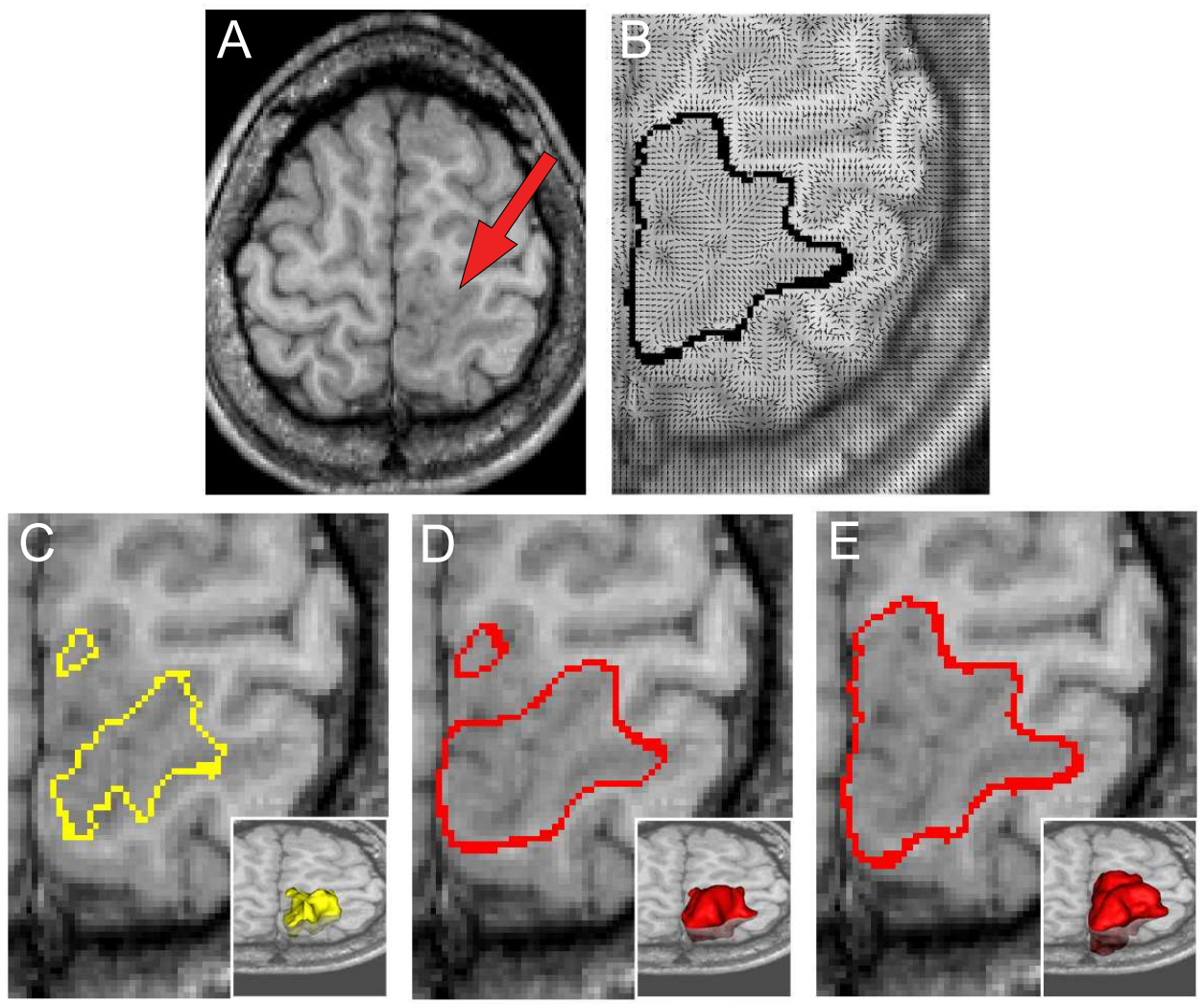

Figure 5: Example of FCD lesion segmentation using the two successive deformable models. (A) T1-weighted MRI where the FCD lesion is indicated by an arrow. (B) Final result shown together with the gradient vector flow $(\mathrm{GVF})$. (C) Initialization with the FCD classifier. (D) Intermediate result of the feature-based deformable model (FDM). (E) Final result using the expansion towards cortical boundaries (ECB). 

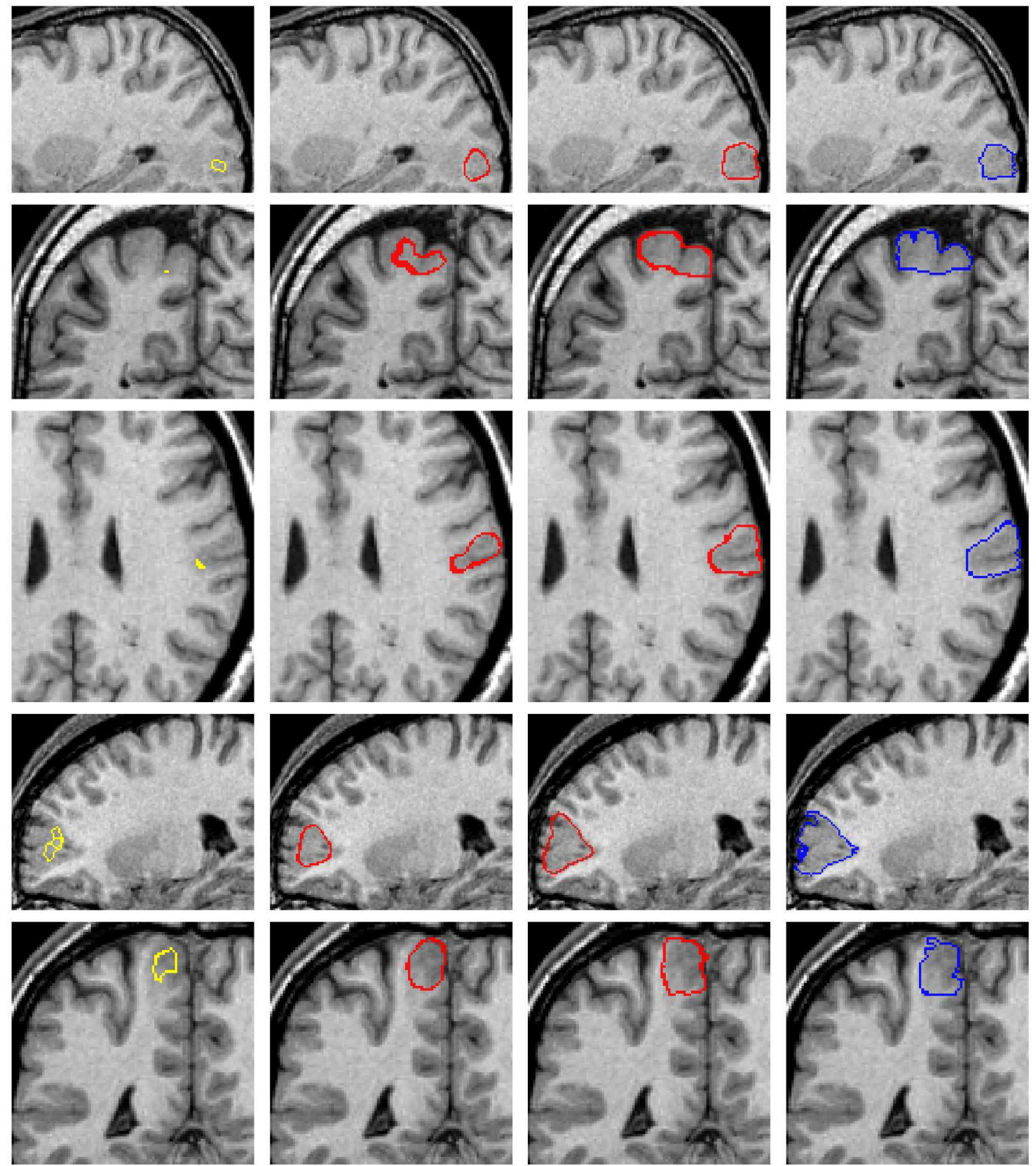

A

B

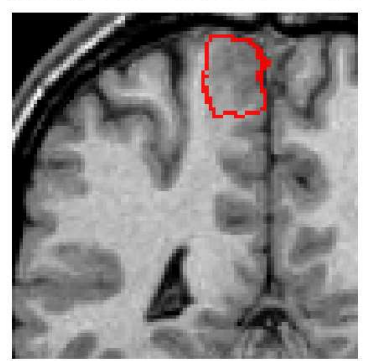

C

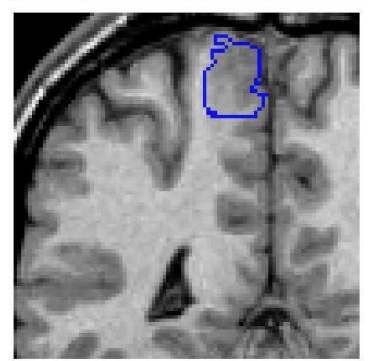

D

Figure 6: Results of the lesion segmentation in five different subjects. Columns show the different steps of the procedure and the corresponding manual label: (A) initialization with the classifier (yellow contour), (B) intermediate result with the FDM (red contour), (C) final result of the automated segmentation with the ECB (red contour), (D) consensus manual label $M_{C}$ (blue contour). 

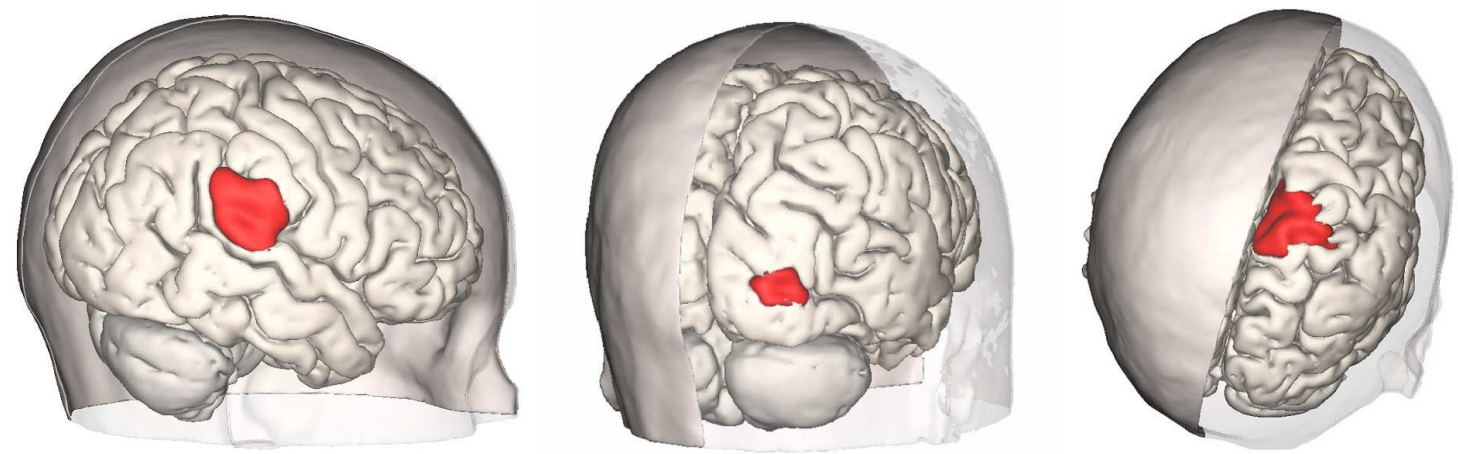

Figure 7: 3D renderings of the automated lesion segmentation in three patients with FCD, shown together with the cortical surface. Visualization was done using the BrainVISA/Anatomist software (www.brainvisa.info). 

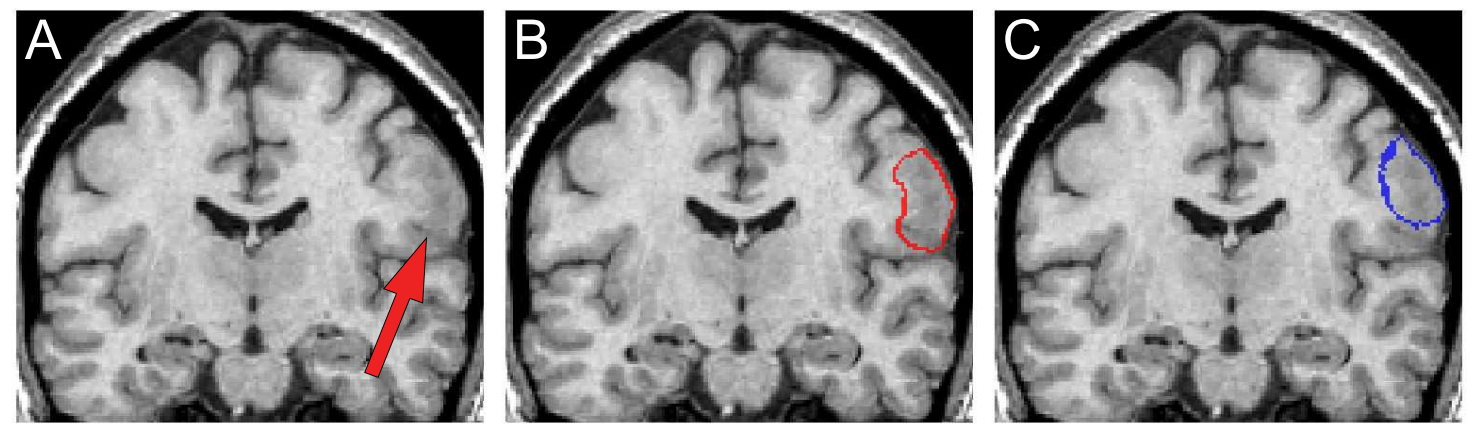

Figure 8: Example of a narrow sulcus which was not correctly extracted. (A) T1-weighted MRI where the sulcus (indicated by the arrow) is located near the inferior boundary of the FCD lesion. (B) Segmentation result. The deformable model has progressed beyond the sulcus resulting in a false positive area. (C) Manually traced lesion label $M_{C}$. 